ISSN 1112-9867

\title{
VACUUM FUSED DEPOSITION MODELLING SYSTEM TO IMPROVE TENSILE STRENGTH OF 3D PRINTED PARTS
}

\author{
S. Maidin ${ }^{1}$, J. H. U. Wong ${ }^{1, *}$, A. S. Mohamed ${ }^{1}$, S. B. Mohamed ${ }^{2}$, R. A. Rashid ${ }^{3}$ and Z. I. \\ Rizman $^{4}$ \\ ${ }^{1}$ Faculty of Manufacturing Engineering, Universiti Teknikal Malaysia Melaka, 76100 Durian \\ Tunggal, Melaka, Malaysia \\ ${ }^{2}$ Faculty of Innovative Design and Technology, Universiti Sultan Zainal Abidin, 21300 Kuala \\ Nerus, Terengganu, Malaysia \\ ${ }^{3}$ Faculty of Languages and Communication, Universiti Sultan Zainal Abidin, 21300 Kuala \\ Nerus, Terengganu, Malaysia \\ ${ }^{4}$ Faculty of Electrical Engineering, Universiti Teknologi MARA, Dungun, Terengganu, \\ Malaysia
}

Published online: 10 November 2017

\begin{abstract}
Functional parts require high a level of strength and the current Fused Deposition Modelling (FDM) cannot be fully utilized as the end used parts. The poor mechanical strength is caused by the incomplete layer bonding during the printing process. In the printing process, the interlayer bonding is made too quick thus the layers are not fully fused together causing the reduced tensile strength. This paper presents a possible solution to this problem by incorporating vacuum technology in FDM system to improve tensile strength of $3 \mathrm{D}$ printed specimens. In this study, a desktop FDM machine was placed and operated inside a low pressure vacuum chamber. The results obtained show an improvement of $12.83 \%$ of tensile strength compared to the standard specimen. This paper concludes that the low pressure environment is useful in reducing the heat loss due to convection of air, hence directly improves the specimen's tensile strength.
\end{abstract}

Author Correspondence, e-mail: ajohn91@live.com

doi: http://dx.doi.org/10.4314/jfas.v9i6s.63 
Keywords: additive manufacturing; fused deposition modelling; vacuum system; mechanical strength.

\section{INTRODUCTION}

Additive Manufacturing (AM) has been around for several decades. The advent of AM technology brings infinite possibilities of designs and products. Fused Deposition Modelling (FDM) is one of the popular and widely used extrusion-based AM technologies. It works by extruding a thin layer thermoplastic filament from a heated small tip nozzle and deposited on a platform in two-dimensional. Upon completion, either the nozzle rises or platform descends to deposit a new layer and bonds with the previous layer creating a 3D solid model [1]. AM is different compared to other conventional technologies where it is capable in producing custom parts within a short time with effective costing. Besides, it is also very flexible when it comes to design and prototyping, offering huge design freedom and complexity [2]. However, FDM possesses weaknesses such as poor mechanical properties [3]. It is nowhere near as strong as parts produced from injection moulding. In fact, the anisotropic behaviour due to layer orientation causes weak level of strength at certain axis [4]. Studies have found that one of the main reasons for poor mechanical strength is the incomplete bonding of layers during layer-by-layer extrusion process. The extruded thin layer of ABS filament at above 200 degrees solidifies too fast for the complete bonding to happen [5-6].

This paper presents a study to integrate a vacuum system with FDM system to address the issue of incomplete bonding of layers during printing process. Vacuum system has been used in various fields of research and all sorts of industries. The ability to create a low to high vacuum pressure range by decreasing the atmospheric pressure allows numerous applications such as drying process, die casting and resin infusion moulding [7-9]. One of the specific traits offered by the vacuum is the reduction of air molecules, which will reduce the convection process by preventing heat to jump from one molecule to another. Heat will be able to be maintained for a longer period of time depending on the vacuum level [10-12].

In this study, a new approach of operating FDM inside a vacuum system was introduced to test its reliability to improve tensile strength. Finite element analysis was used to observe the thermal flow in different pressure environment. This is followed by five experimental tests with each three specimens was conducted at 5 different vacuum levels, 30, 27, 24, 21 and 18 inHg respectively. The variables which include the material (ABS), layer height $(0.3 \mathrm{~mm})$, temperature $\left(260^{\circ} \mathrm{C}\right)$ and infill density were kept constant. The specimens were oriented 
vertically at its weakest state to test its bonding strength. After the tensile tests were completed, the maximum stress for each test was recorded and tabulated.

\subsection{Fused Deposition Modelling}

FDM is an extrusion based system from additive manufacturing technology. Operated around wide range of polymer based material, FDM is one of the widely accessible and used technologies in the world. In late 1980s, Scott Crump, one of the co-founders of Stratasys developed FDM system by mechanically extruding molten polymer layer by layer and was commercialized later on [13-14]. Fused deposition modelling operates by saving a 3D CAD drawing data into an STL format. The FDM software then reads the STL file for which the user can adjust the parameters (layer height, speed, infill \% and etc.). Upon accepting the parameters set, the software slices the model into layers and generates support if required. It is ready to draw a flexible filament from the spool/compartment into a heated nozzle via a geared motor at constant temperature value based on the materials selected. The extruded thin filament from the nozzle tip is deposited onto a build platform in two dimensional ( $\mathrm{x}$ and $\mathrm{y}$ ) axes. The nozzle or platform moves in z-axis to allow a new layer to deposit onto the previous built layer. The deposited layers are then solidified and left to be cooled down. The process is repeated until all the layers are deposited completely and form a solid object [15].

FDM is evolving rapidly and it offers immense advantages. Unlike traditional subtractive manufacturing, FDM produces minimal waste since the raw materials are efficiently used by extruding layer by layer [31]. FDM requires little manufacturing stages hence consuming low energy [16]. Besides, FDM is able to produce customized parts quickly and cheaper than conventional manufacturing. Furthermore, FDM is also able to produce difficult and complex parts which are deemed difficult for conventional manufacturing [17]. Despite having numerous advantages, FDM also possesses several weaknesses, such as poor mechanical properties. Mechanical strength is one of the most discussed problems among FDM users and researchers. FDM produced part inhibits anisotropic behaviour. The physical properties are dependent on the build orientation for which the mechanical test will differ based on the direction of stress is exerted [18]. Various methods have been introduced to improve the strength of FDM produced parts. One of the easiest ways is to modify build parameters such as increasing the infill percentage and number of shells and orientating the best possible way so that the parts are at their strongest points [19-20]. However, it is time consuming and not every complex shape can be orientated correctly when there are two or more forces acting on different axis. Previous studies have also introduced several other materials that can be used to improve the strength of FDM parts compared to the commonly used ABS and PLA [21-22]. 
Compositing with high strength resin into the void of the FDM parts also increases its mechanical strength. Other than that, chemical treatment can be used as one of the alternatives to improve the parts' strength [23]. One of the reasons for poor strength is the incomplete bonding of layers. During extrusion of molten thermoplastic layer on top of the previous layer, it is the mechanical adhesions that occur instead of chemical adhesions. The rapid drop of filament temperature from the nozzle to room temperature causes partial bonding as well [24].

\subsection{Vacuum Technology}

Vacuum is an empty or void space of matter where there is an absence of particles. Perfect vacuum is impossible to be achieved in the laboratory. Hence the word vacuum is generally defined as a region or space with gaseous pressure lower than atmospheric pressure [25]. Normally, the atmospheric pressure at 30 inHg contains air molecules that are constantly hitting one another. By reducing the air molecules, a wide range of applications can be used in research areas and industrial sectors. Vacuum can range from low to ultra-high level. At a particular atmospheric pressure, the molecules keep bombarding to transmit energy from one place to another. The higher the molecules' density, the easier the transmission of energy occurs. The molecules are relatively closer to one another thus hitting in every direction [26]. However, if the air molecules are reduced, there is less medium to transfer the energy around. On the contrary, the change of physical properties of air from the change of vacuum pressure also affects different thermal behaviour. Heat loss due to convection can be reduced by the absence of air particles [27].

\subsection{Transfer of Heat Energy}

Energy is a property that exists in many forms and it involves in transferring work to alter the state of the substances [28]. Come in many forms, one of the useful energies in real applications is thermal energy or known as heat energy. The flow of heat energy occurs when there is a difference in temperature between two bodies and it stops when the two bodies reach equilibrium temperature. Heat energy is interrelated with temperature. As the temperature increases, heat energy increases and vice versa. Even though both may be alike, they are not the same [29]. For example, thermoplastic needs more heat energy than steel to rise towards a specific temperature since both have different heat conductivities. Thus, temperature is affected by the amount of heat energy and the nature of the material. Heat transfer may occur through conduction, convection and radiation. Inside the vacuum system depending on the vacuum level, convection still exists. Radiation still occurs since it does not require any medium to travel. Conduction will take place just within the FDM machine. Convection occurs where there is a liquid or gaseous medium for heat energy to travel. In a 
natural convection state, the motion of fluid occurs when there is a change in density of fluid or gases. Heat transfer for vertical plates in equation 1 can be conducted with accuracy of RA > $10^{9}$.

$$
\overline{\mathrm{N}} \mathrm{u}_{\mathrm{L}}=\left\{0.825+\frac{0.387 \mathrm{Ra}_{\mathrm{L}}{ }^{1 / 6}}{\left[1+\left(\frac{0.492}{\mathrm{Pr}}\right)^{9 / 16}\right]^{8 / 27}}\right\}^{2}
$$

Convention only happens when there is a medium for it to travel. If there is no medium exists through absolute vacuum, heat cannot be transferred by any means except for radiation. However, for practical applications in real life, heat transfer through convection can still be reduced subject to the degree of vacuum level.

\section{METHODOLOGY}

\subsection{Finite Element Analysis(FEA)}

FEA was conducted to study the behaviour of heat transfer in the normal and the vacuum environments. SolidWorks Flow Simulation was used to create a virtual vacuum environment inside a chamber and to generate heat source. The aim of this simulation was to observe the movement of air molecules within the gases or known as convective heat transfer. An enclosed chamber with $350 \times 390 \times 400 \mathrm{~mm}$ was used as internal analysis and any external factors were disregarded. Inside the chamber, there were a nozzle $\left(260^{\circ} \mathrm{C}\right)$ and heat bed $\left(100^{\circ} \mathrm{C}\right)$ emitting constant heat temperature as shown in Table 1 . The simulation was carried for 1 hour with 1 minute time step interval. Gravity of $-9.81 \mathrm{~m} / \mathrm{s}^{2}$ was present and the initial temperature was set at $20^{\circ} \mathrm{C}$. Different pressures ranged at 30,20 and $10 \mathrm{inHg}$ were used for the simulation study. The air velocity was in steady state with laminar and turbulent air flow. The cut plots were shown in Fig. 1a, Fig. 1b and Fig. 1c. 
Table 1.Simulation input data

\begin{tabular}{cc}
\hline Input & Value \\
\hline Simulation time & $3600 \mathrm{sec}$ \\
Time step interval & $60 \mathrm{sec}$ \\
Gravity & $-9.81 \mathrm{~m} / \mathrm{s}^{2}$ \\
Air pressure & $30,20,10 \mathrm{inHg}$ \\
Air velocity & Steady state \\
Air type & Laminar and turbulent \\
Wall condition & Adiabatic wall \\
Initial temperature & $20.05^{\circ} \mathrm{C}$ \\
Constant nozzle/bed temperature & $260^{\circ} \mathrm{C} / 100^{\circ} \mathrm{C}$ \\
\hline
\end{tabular}
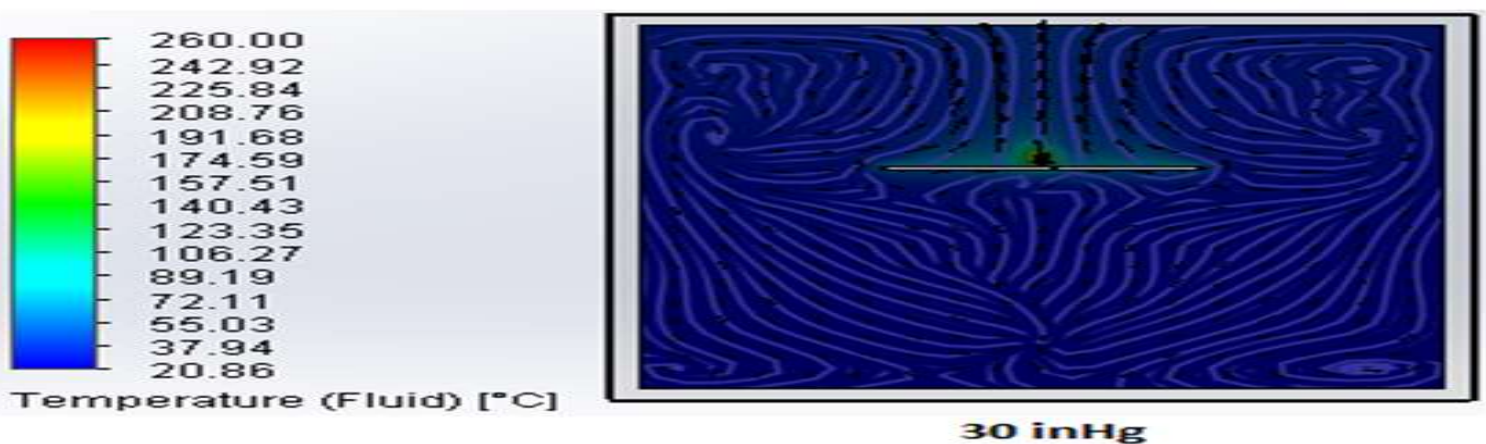

Fig.1a.Contours, vectors and streamlines on $30 \mathrm{inHg}$
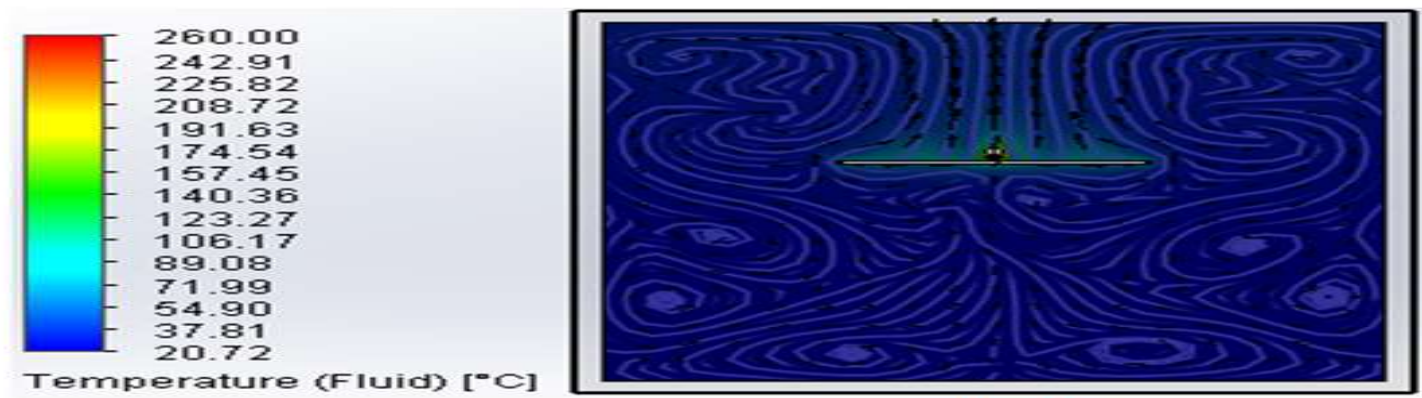

20 inHg

Fig.1b.Contours, vectors and streamlines on $20 \mathrm{inHg}$ 

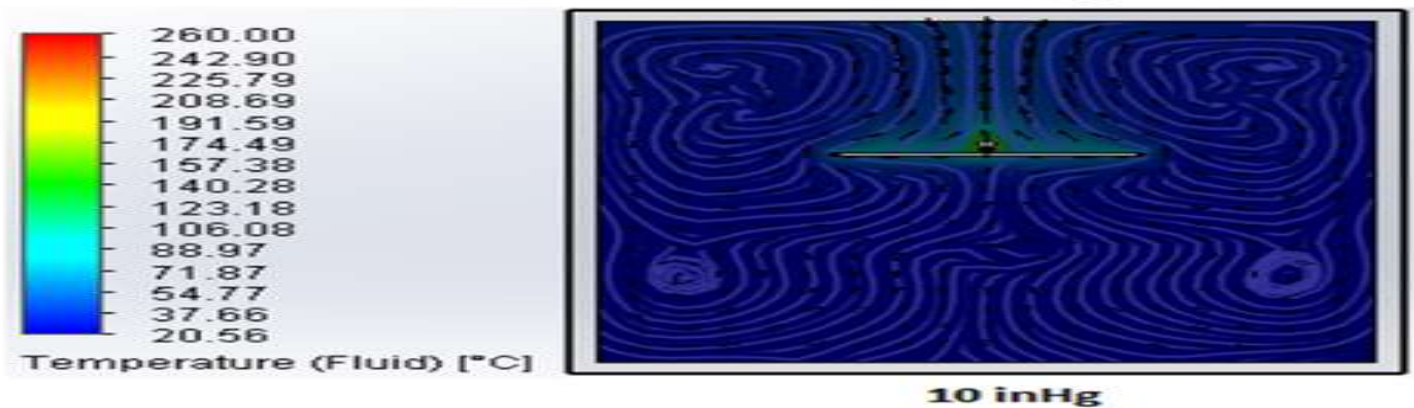

Fig.1c. Contours, vectors and streamlines on $10 \mathrm{inHg}$

The air flow was similar across the three simulations. The hot air dispersed upwards from the heat source and slowly moved outwards and downwards which was caused by the density of air molecules. However, the patterns were different. At $30 \mathrm{inHg}$, the free mean path was relatively small and the molecules collided with each other. The lines were irregular and not parallel. Random movements were also observed. At $20 \mathrm{inHg}$, the molecules flow began to reconstruct from turbulent flow in a viscous state. At $10 \mathrm{inHg}$, laminar flow was observed and the patterns were much more regular compared to $30 \mathrm{inHg}$. By comparing the three flows, the lowest minimum temperature was found at $10 \mathrm{inHg}$ showing $20.56^{\circ} \mathrm{C}$ and the highest minimum temperature was at $30 \mathrm{inHg}$ showing $20.86^{\circ} \mathrm{C}$. The simulations proved that convective heat transfer can be reduced by dropping the pressure. In order to strengthen the hypothesis, an actual experiment was conducted.

\subsection{Experimental Setup}

A vacuum system suitable for the Up Plus 2 FDM machine was fabricated to determine the feasibility of vacuum in improving the strength of the produced specimens (see Figs. 2a and 2b). Made from acrylic, the chamber's dimension was $350 \times 390 \times 400 \mathrm{~mm}$ with $12 \mathrm{~mm}$ thickness.

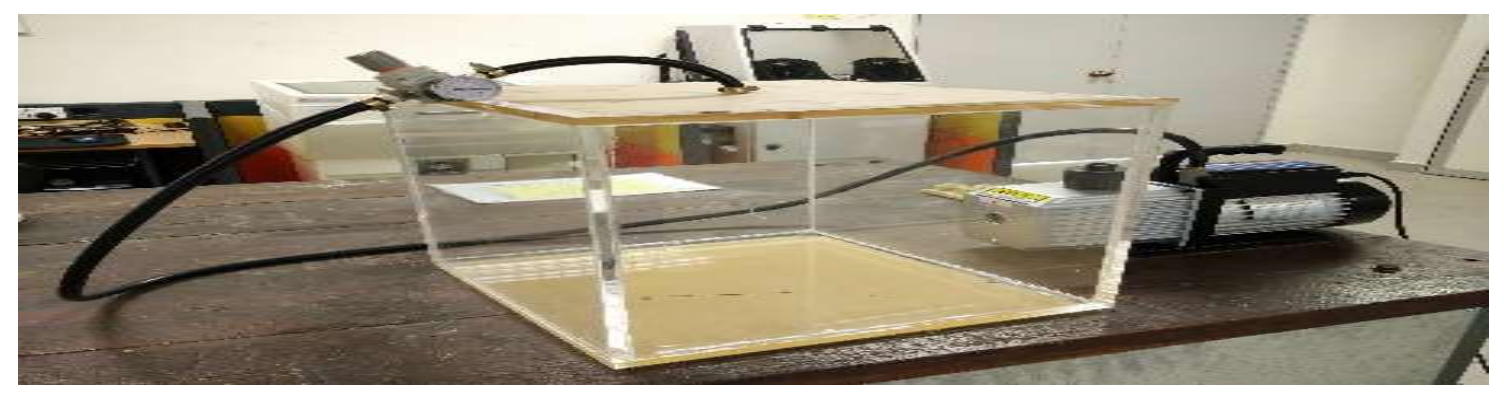

Fig.2a. Fabricated vacuum chamber and the assembly of the whole system 


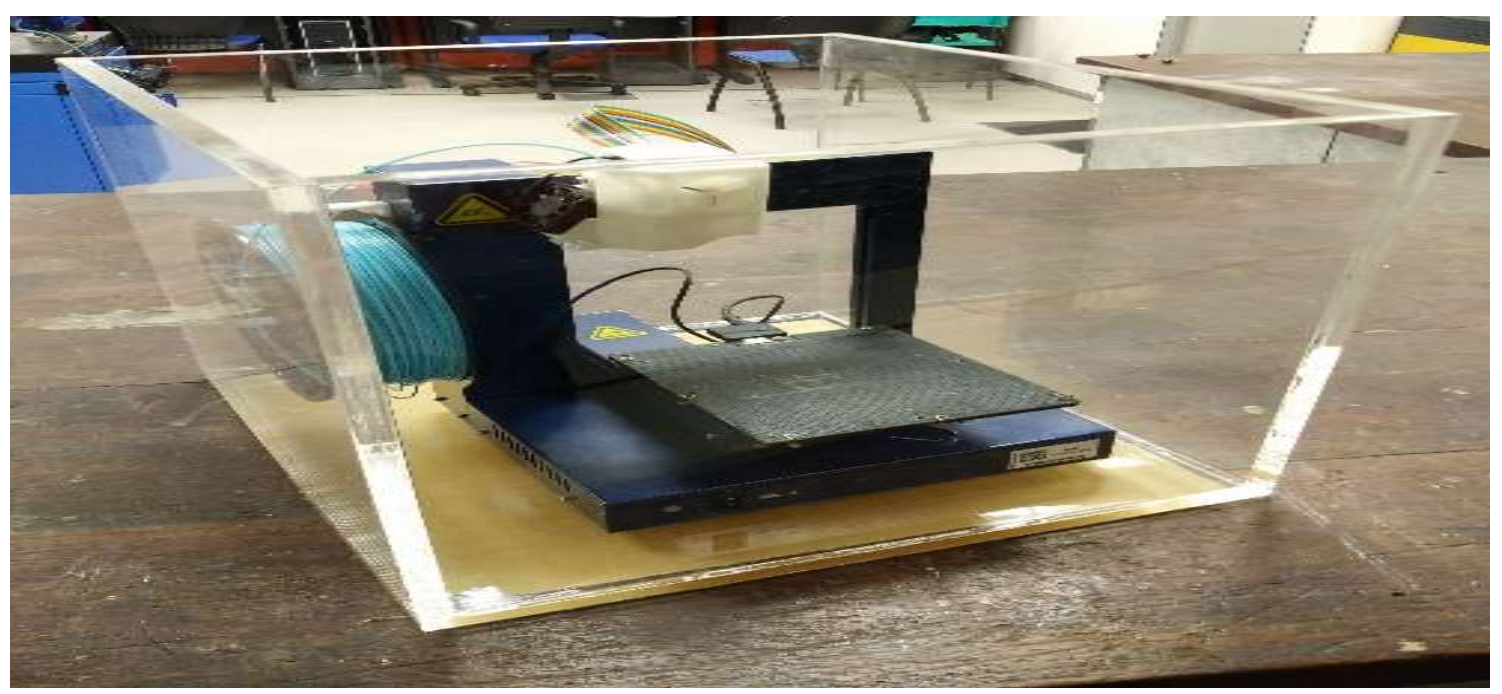

Fig.2b. The placement of Up Plus 2 FDM machine into the chamber

The specimens were modelled in SolidWorks software and saved as STL format. The STL was imported into Up Plus 2 dedicated software. The factors were set as per design of the parameters given. Prior to the FDM operations, the machine had been calibrated to ensure consistency through the whole experiments. There were two main calibrations required: platform levelling and height levelling. After calibration, the specimens were produced at 30, 27, 24, 21 and $18 \mathrm{inHg}$ vacuum pressure. The layer thickness was set at $0.30 \mathrm{~mm}$ with constant heat bed temperature at $100^{\circ} \mathrm{C}$ and nozzle $260^{\circ} \mathrm{C}$. The specimens used were according to ASTM D638 Type 4 having dimensions $115 \mathrm{~mm}$ x $19 \mathrm{~mm}$ x $3.4 \mathrm{~mm}$. The specimens were orientated to be printed in z-direction in order to test its bonding strength, as shown in Fig. 3. The produced specimens were labelled and kept in separate plastic seals. 


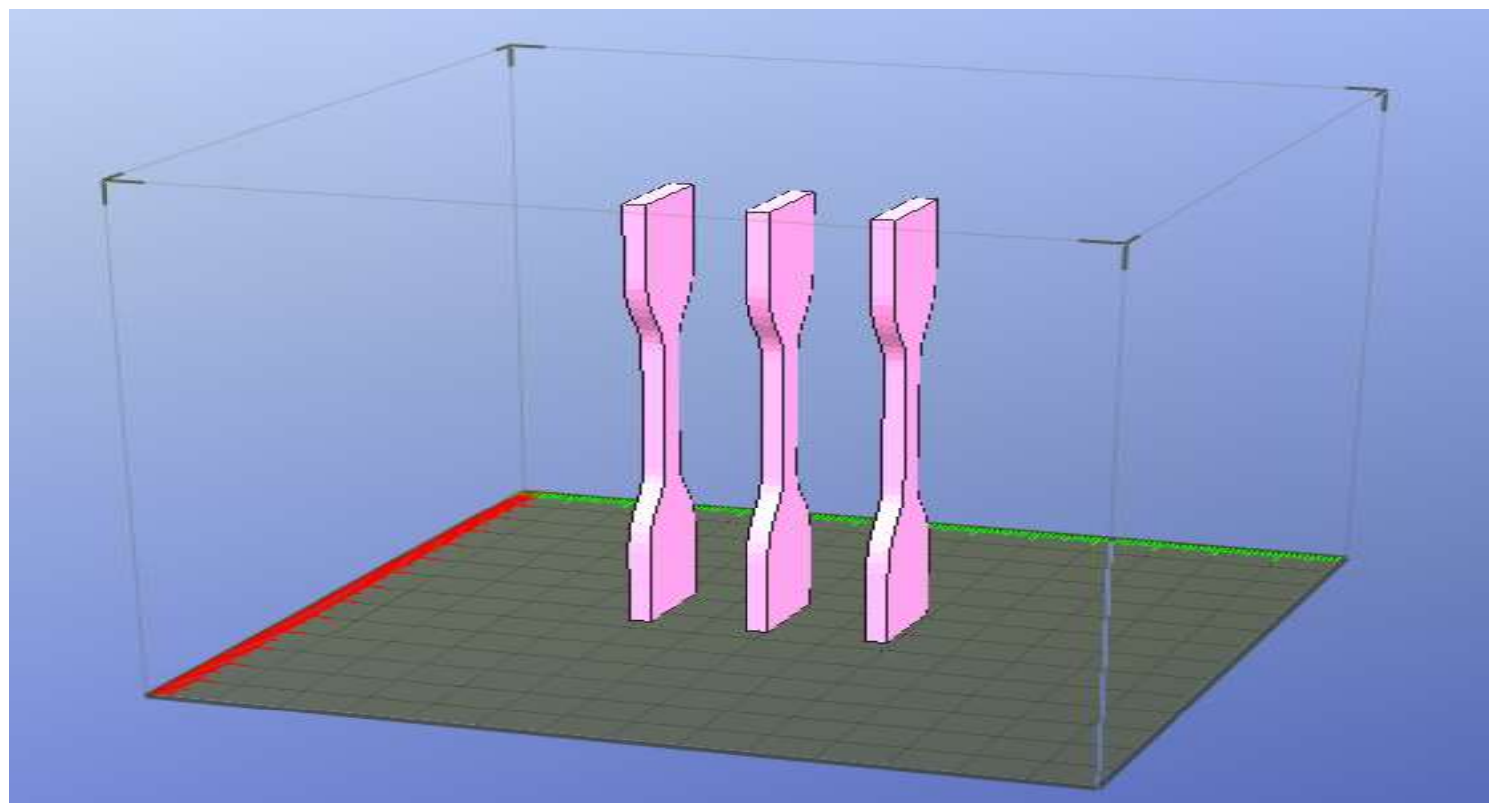

Fig.3. Specimens were orientated vertically

A total of five sets of ABS specimens were produced based on the vacuum level as shown in Fig. 4.

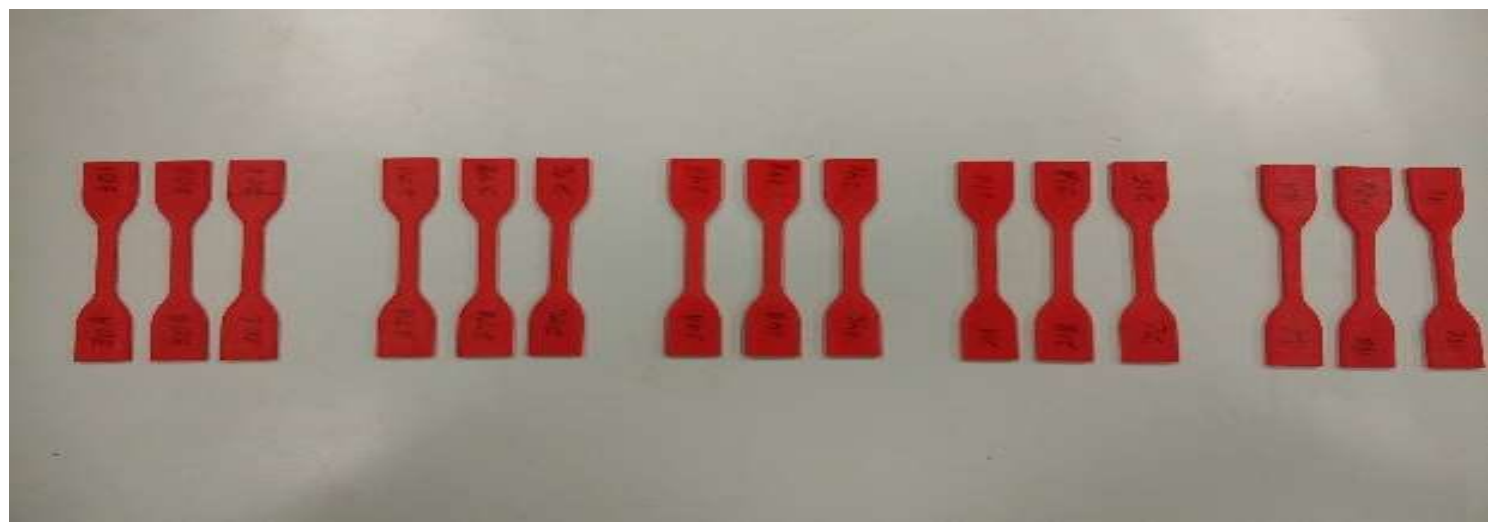

Fig.4. Specimens produced at different vacuum levels: 30, 27, 24, 21 and $18 \mathrm{inHg}$

\subsection{Specimen Test}

After printing all the specimens from the FDM machine, they were kept sealed to prevent moisture and dirt from affecting them. During tensile test, potential unwanted factors were carefully controlled [32] to ensure the accuracy of the results. Tensile test at break was chosen based on ASTM D638, Standard Test Method for Tensile Properties of Plastics. The UTM machine used to carry the test was Autograph Universal Testing Machine with Trapezium software. The force was $10 \mathrm{kN}$, cross head speed at $5 \mathrm{~mm} / \mathrm{min}$. After each test, the data and graph were saved. The broken specimens were kept and observed under scanning electron microscope (SEM) to examine their microstructures. Fig. 5 shows the universal tensile machine used for tensile test. 


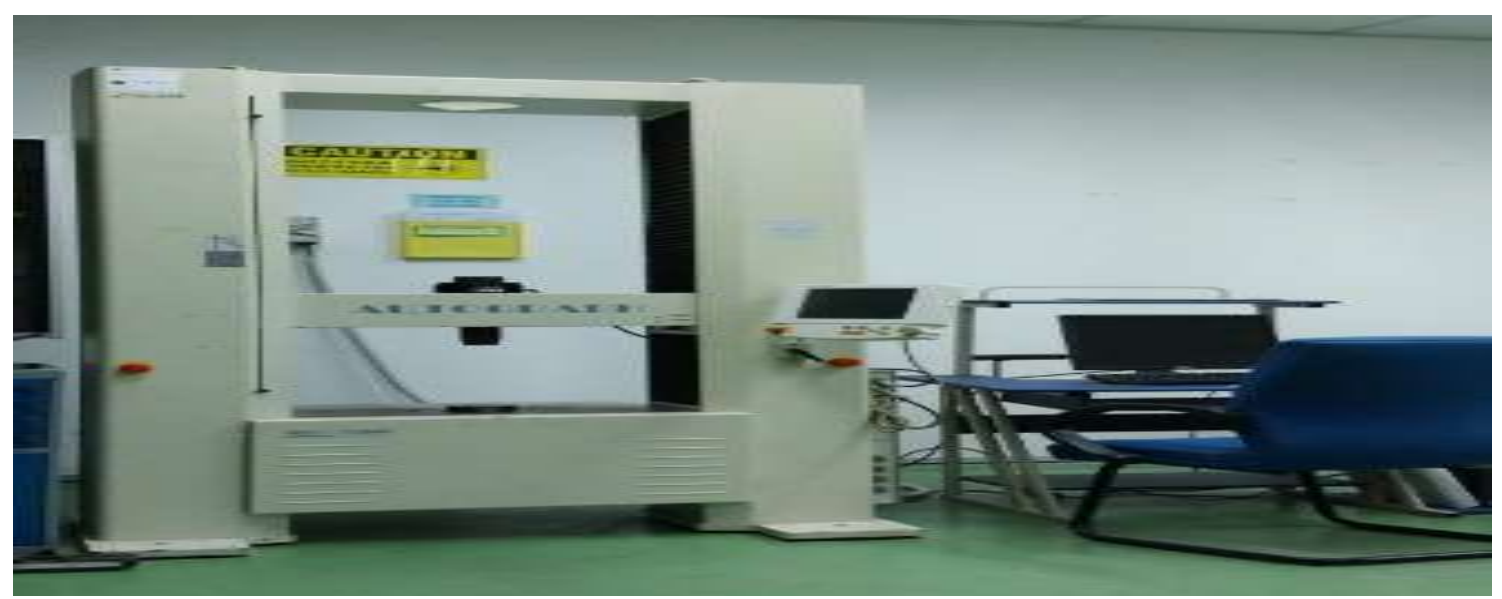

Fig.5.Autograph universal testing machine

\section{RESULTS AND DISCUSSION}

Tensile test was conducted to determine the effect of vacuum system on the FDM specimens' mechanical strength. The results are tabulated as follow.

Table 2.Maximum Tensile Strength for ABS Specimens

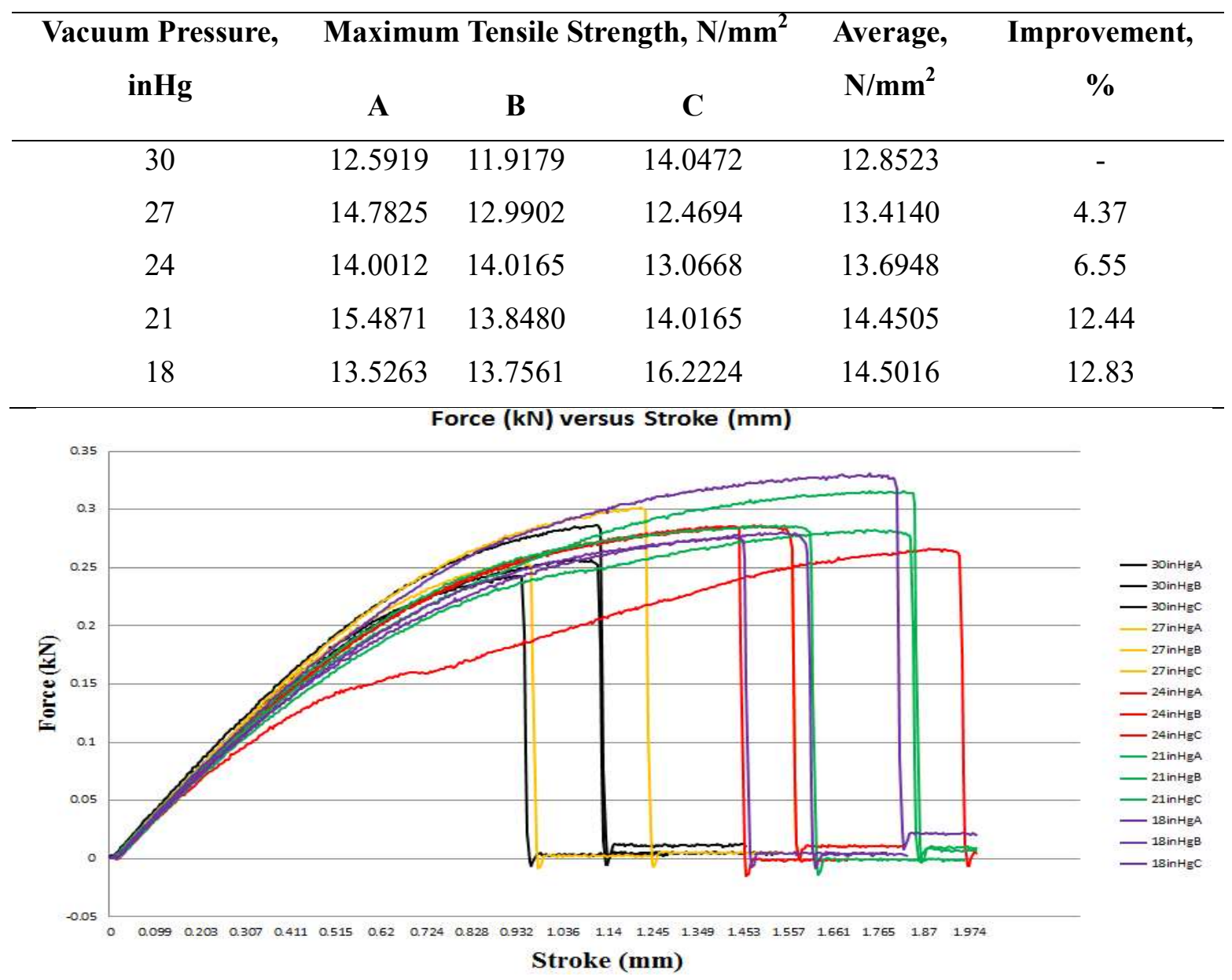

Fig.6. Force $(\mathrm{kN})$ vs stroke $(\mathrm{mm})$ of ABS specimens tested 
Based on the data presented in Table 2 and Fig. 6, it can be clearly seen that maximum tensile strength increases as the vacuum pressure increases. The standard atmospheric level for the starting point and benchmark of this experiment is $30 \mathrm{inHg}$ or 1 atmosphere. Overall, the specimens produced under $18 \mathrm{inHg}$ had the highest maximum tensile strength at 14.5016 $\mathrm{N} / \mathrm{mm}^{2}$ compared to $30 \mathrm{inHg}(1 \mathrm{~atm})$ at $12.8523 \mathrm{~N} / \mathrm{mm}^{2}$ with the improvement of $12.83 \%$. The vacuum pressure began at below $30 \mathrm{inHg}$. At the beginning of vacuum $27 \mathrm{inHg}$, the specimens were able to sustain higher force during tensile test giving $4.37 \%$ increment. The same applied to $24 \mathrm{inHg}$ with the pressure progressively decreased, giving $6.55 \%$ improvement. Starting from $21 \mathrm{inHg}$, the tensile strength increased dramatically to $12.44 \%$ improvement which is a better value compared to $24 \mathrm{inHg}$. At the last vacuum pressure of 18 inHg, the percentage of improvement increased steadily to $12.83 \%$. Based on these results, it can be concluded that the use of vacuum system changed the environment where the FDM was operating and improved the mechanical strength of the FDM specimens. The broken specimens from tensile test were observed under SEM as shown in Fig. 7a and Fig. 7b. At 30 inHg, more pores were observed. There were cracks, shallow and deep pits at several places mainly due to distortion and stress during layer bonding formation. The hot semi-molten thermoplastic expands when heated and sudden contracts caused the poor bonding. At 18 inHg, fewer pores were observed and the surface was clean. The specimens were not broken at the same layer, but combined together with the adjacent layer. Therefore, the bonding was strong at holding the layers together before it broke during tensile test.

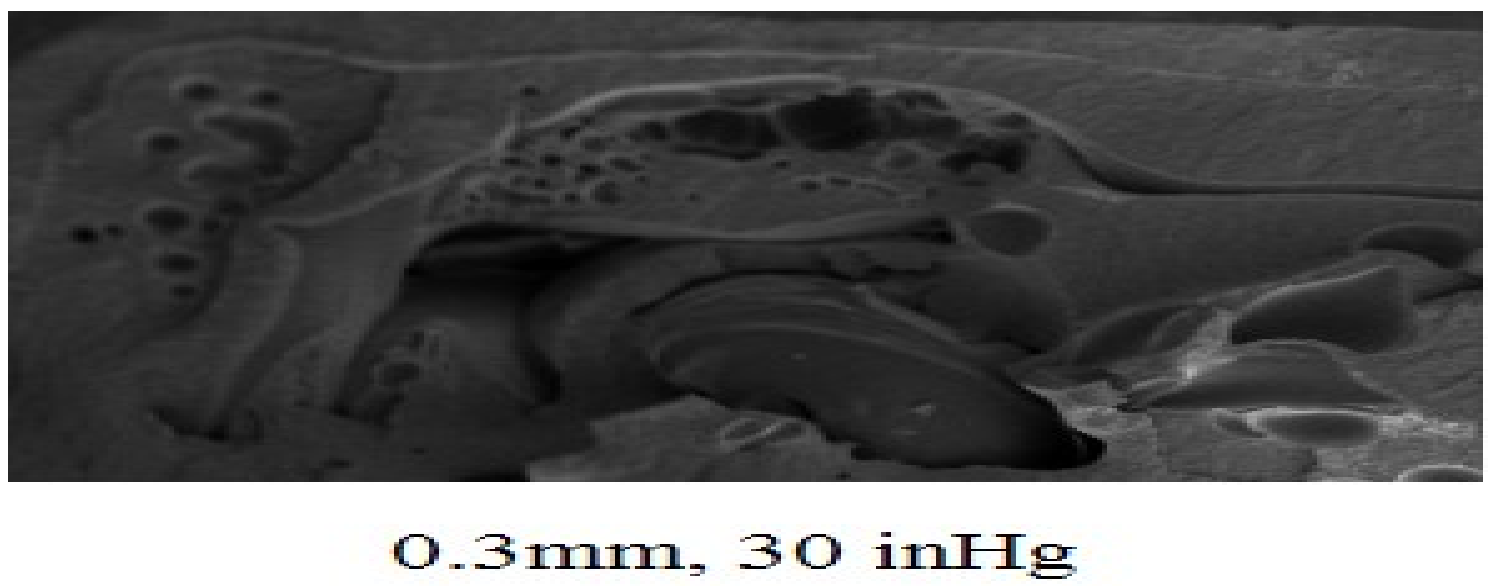

Fig.7a. SEM image of microstructures at $30 \mathrm{inHg}$ 


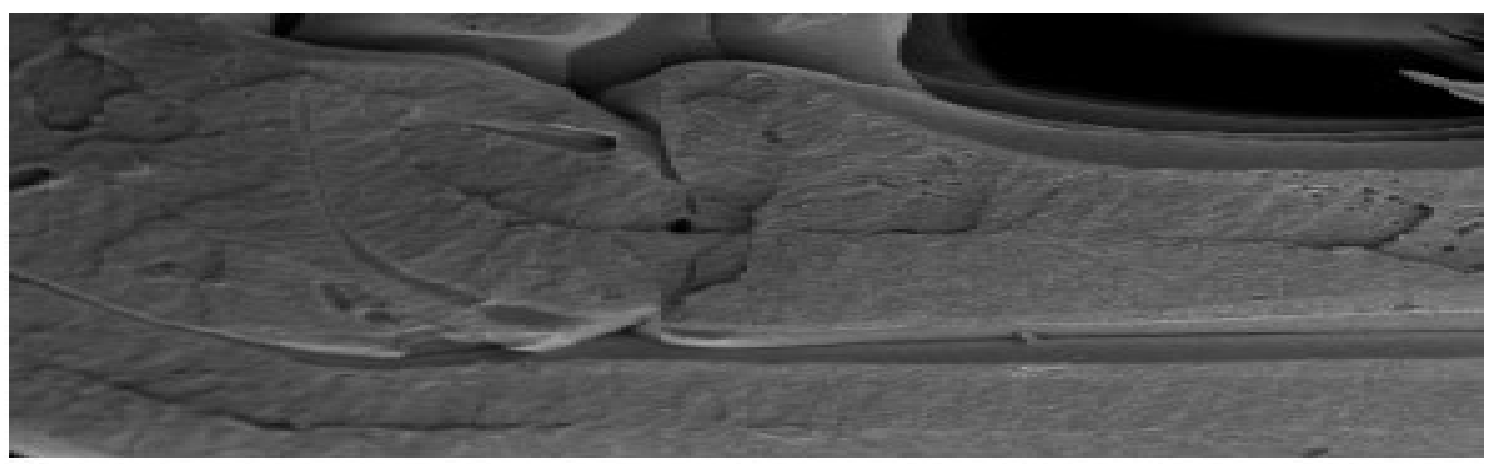

\section{$0.3 \mathrm{~mm}, 1 \mathrm{~S} \mathrm{inFIg}$}

Fig.7b. SEM image of microstructures at $18 \mathrm{inHg}$

\section{CONCLUSION}

This study aimed to identify a possible and better way to improve fused deposition modelling part's mechanical strength [30]. The vacuum assisted FDM was proven to be effective by creating a low pressure environment for the FDM process. In vacuum state, the density of air molecules was less compared to the one atmospheric state. Hence, convection of heat energy was harder to take place. Once extruded from the nozzle, the heat remained at the semi-molten thermoplastic for a longer period of time. Rapid cooling can be eliminated which contributed to stress and distortion. The bonding formation from the thermal energy of semi-molten ABS material between each layer was more effective and better compared to the non-vacuum. Although increasing the extruder temperature helped to produce better bonding of layers, the part's accuracy and surface quality were undesirable. There is a drawback of using vacuum system onto the FDM system. The electronics are affected by the heat trapped inside the chamber. Continuous use of the FDM may damage the built-in components. Therefore, it is suggested that the FDM system is redesigned with the electronics placed out leaving the mechanical system of FDM inside the vacuum system. For future research, higher vacuum pressure can be explored to determine its effect on mechanical properties as well as other properties.

\section{ACKNOWLEDGEMENTS}

The authors would like to acknowledge the Universiti Teknikal Malaysia Melaka (UTeM) for the scholarship of 'Skim Zamalah UTeM' and the Ministry of Higher Education Malaysia for awarding the Fundamental Research Grant Scheme (FRGS) grant number FRGS/1/2015/TK03/FKP/02/F00282. 


\section{REFERENCES}

[1] Hossain MS, Ramos J, Espalin D, Perez M, Wicker R. Improving tensile mechanical properties of FDM-manufactured specimens via modifying build parameters. InInternational Solid Freeform Fabrication Symposium: An Additive Manufacturing Conference, pp. 380-392

[2] Onwubolu GC, Rayegani F. Characterization and optimization of mechanical properties of ABS parts manufactured by the fused deposition modelling process. International Journal of Manufacturing Engineering, 2014, 2014:1-13

[3] Belter J T, Dollar A M. Strengthening of 3D printed fused deposition manufactured parts using the fill compositing technique. PloS One, 2015, 10(4):1-19

[4] Nelaturi S, Shapiro V. Representation and analysis of additively manufactured parts. Computer-Aided Design, 2015, 67:13-23

[5] Sun Q, Rizvi G M, Bellehumeur C T, Gu P. Effect of processing conditions on the bonding quality of FDM polymer filaments. Rapid Prototyping Journal, 2008, 14(2):72-80

[6] Bellehumeur C, Li L, Sun Q, Gu P. Modeling of bond formation between polymer filaments in the fused deposition modeling process. Journal of Manufacturing Processes, 2004, 6(2):170-178

[7] Criscuoli A, Carnevale M C, Drioli E. Study of the performance of a membrane-based vacuum drying process. Separation and Purification Technology, 2016, 158:259-265

[8] Kapranos P, Brabazon D, Midson S P, Naher S, Haga T. Advanced casting methodologies: Inert environment vacuum casting and solidification, die casting, compocasting, and roll casting. Comprehensive Materials Processing, 2014, 5:3-37

[9] Gu Y, Tan X, Yang Z, Zhang Z. Hot compaction and mechanical properties of ramie fabric/epoxy composite fabricated using vacuum assisted resin infusion molding. Materials and Design. 2014, 56:852-861

[10]Loo W. Y., Loo K. W. Physics insights 'O' level. Jurong: Pearson Longman, 2007

[11]Avison J. The world of physics.England: Nelson Thornes, 2014

[12]Chambers A. Modern vacuum physics.Florida: CRC Press, 2004

[13]Gao W, Zhang Y, Ramanujan D, Ramani K, Chen Y, Williams C B, Wang C C, Shin Y C, Zhang S, Zavattieri P D. The status, challenges, and future of additive manufacturing in engineering. Computer-Aided Design, 2015, 69:65-89

[14]Stratasys. $\quad$ FDM technology. 2017, http://www.stratasys.com/3d-printers/technologies/fdm-technology 
[15]Lee C S, Kim S G, Kim H J, Ahn S H. Measurement of anisotropic compressive strength of rapid prototyping parts. Journal of Materials Processing Technology, 2007, 187:627-630

[16]Ivanova O, Williams C, Campbell T. Additive manufacturing (AM) and nanotechnology: Promises and challenges. Rapid Prototyping Journal, 2013, 19(5):353-364

[17]Ziemian C, Sharma M, Ziemian S. Anisotropic mechanical properties of ABS parts fabricated by fused deposition modelling.Rijeka: INTECH Open Access Publisher, 2012

[18]Hildebrand K, Bickel B, Alexa M. Orthogonal slicing for additive manufacturing. Computers and Graphics, 2013, 37(6):669-675

[19]Torrado A R, Shemelya C M, English J D, Lin Y, Wicker R B, Roberson D A. Characterizing the effect of additives to $\mathrm{ABS}$ on the mechanical property anisotropy of specimens fabricated by material extrusion 3D printing. Additive Manufacturing, 2015, 6:16-29

[20]Raut S, Jatti V S, Khedkar N K, Singh T P. Investigation of the effect of built orientation on mechanical properties and total cost of FDM parts. Procedia Materials Science, 2014, 6:1625-1630

[21]Bagsik A, Schöppner V, Klemp E. FDM part quality manufactured with Ultem* 9085. In 14th International Scientific Conference on Polymeric Materials, 2010, pp. 307-315

[22]Nikzad M, Masood S H, Sbarski I. Thermo-mechanical properties of a highly filled polymeric composites for fused deposition modeling. Materials and Design, 2011, 32(6):3448-3456

[23]Galantucci L M, Lavecchia F, Percoco G. Quantitative analysis of a chemical treatment to reduce roughness of parts fabricated using fused deposition modeling. CIRP Annals-Manufacturing Technology, 2010, 59(1):247-250

[24]Sood A K, Ohdar R K, Mahapatra S S. Improving dimensional accuracy of fused deposition modelling processed part using grey Taguchi method. Materials and Design, 2009, 30(10):4243-4252

[25] Shao H, Jiang S, Zhang X, Wu Z, Wang K, Zhang W. Influence of vacuum degree on the effect of gas explosion suppression by vacuum chamber. Journal of Loss Prevention in the Process Industries, 2015, 38:214-223

[26]O'Hanlon J. F. A user's guide to vacuum technology.New Jersey: John Wiley and Sons, 2005

[27]Achenani Y, Saâdaoui M, Cheddadi A, Bonnefont G, Fantozzi G. Finite element modeling of spark plasma sintering: Application to the reduction of temperature inhomogeneities, case of alumina. Materials and Design. 2017, 116:504-514 
[28]Moss K. J. Heat and mass transfer in buildings.England: Routledge, 2015

[29]Beard M. Environmental chemistry in society.Florida: CRC Press, 2013

[30]Ibrahim Z, Mohamed S B, Minhat M, Mohamed A S, Musanih M R, Rahman Z A, Rizman Z I. Reason maintenance in product modelling via open source CAD system. International Journal on Advanced Science, Engineering and Information Technology, 2016, 6(6):990-996

[31]Minhat M, Mohamed S B, Kasim M S, Sulaiman M A, Rizman Z I. Advanced manufacturing of an aircraft component (fish-head): A technology review on the fabrication. International Journal on Advanced Science, Engineering and Information Technology, 2016, 6(5):734-739

[32]Mohamed S B, Minhat M, Kasim M S, Adam M H, Sulaiman M A, Rizman Z I. An intelligent lighting control system (ILCS) using Labview. Journal of Fundamental and Applied Sciences, 2017, 9(2S):602-615

\section{How to cite this article:}

Maidin S, Wong J U H, Mohamed A S, Mohamed S B, Rashid R A, Rizman Z I. Vacuum fused deposition modelling system to improve tensile strength of $3 \mathrm{~d}$ printed parts. J. Fundam. Appl. Sci., 2017, 9(6S), 839-853. 